\title{
Multiple Koszul structures on lines and instanton bundles
}

\author{
Thomas Nüßler Günther Trautmann \\ March 1993 \\ Dedicated to the memory of Constantin Banica
}

\section{Contents}

Introduction

1 Multiple extensions and Koszul structures on lines

2 The Beilinson resolution

3 Instanton bundles with one linear section

4 Extensions of Koszul structures and elementary transformations

5 Normal forms of monad arrows 


\section{Introduction}

In this paper we prove that the moduli scheme $M I(n)$ of mathematical instanton bundles over $\mathbb{P}_{3}$ with second Chern class $n$ is smooth at bundles $\mathcal{E}$ with $h^{0} \mathcal{E}(1) \neq 0$. By a result in [6] this number is $\leq 2$. In case $h^{0} \mathcal{E}(1)=2$ of special 't Hooft bundles the smoothness is a result of A. Hirschowitz-M.S. Narasimhan in [8]. This case is included in our proof. In the remaining general situation $h^{0} \mathcal{E}(1)=1$, the reduced zero scheme $Z_{\text {red }}$ of the unique section is a disjoint union of lines, but the scheme $Z$ itself has nilpotent structure in general. Here we cannot deform the scheme $Z$ as a zero scheme. We study these nilpotent structures, which turn out to have resolutions

$$
0 \rightarrow n \mathcal{O}(-2) \rightarrow 2 n \mathcal{O}(-1) \rightarrow n \mathcal{O} \rightarrow \mathcal{O}_{Z} \rightarrow 0
$$

given by nice regular matrices, and which are self-dual, see 1.1. We call such structures of Koszul type or Koszul structures. They are exactly the primitive structures of type $\mathcal{O}_{\ell}$ in the notation of C. Banica- O. Forster, [5], see Corollary 1.9.

In proposition 2.3 we show that a multiple structure $X$ on a line, which admits a resolution of the above type with unspecified matrices, and which satisfies $\omega_{X}=$ $\mathcal{O}_{X}(-2)$, is already a Koszul structure given by special matrices as in 1.2 . It follows from this that the zero scheme $Z$ of the unique section of $\mathcal{E}(1)$ as above is a disjoint union of Koszul structure. This enables us to prove $H^{1} \mathcal{N}_{Z}^{\mathrm{V}}(1)=0$ for the conormal sheaf of $Z$, which in turn implies $\operatorname{Ext}^{2}(\mathcal{E}, \mathcal{E}(-1))=0$ and $E x t^{2}(\mathcal{E}, \mathcal{E})=0$, and hence smoothness of $M I(n)$ at $\mathcal{E}$.

The Koszul structures had occured already implicitly in [10] in the description of elementary transformations of instanton bundles along lines. It is remarkable, see section 4, that extensions of Koszul structures are closely related to these elementary transforms. The paper is closed by presenting a normal form for the right part of the monad of an instanton bundle $\mathcal{E}$ with $h^{0} \mathcal{E}(1)=1$ and a single multiple line as its zero scheme.

It turned out that the statements about Koszul structures generalize to higher dimensional linear projective subspaces. This will be treated in a separate note.

Acknowledgement: We thank the DFG-Schwerpunkt "Komplexe Mannigfaltigkeiten" for support. 


\section{Notation}

- $k$ denotes an algebraically closed ground field of characteristic 0 .

- $\mathbb{P}_{3}=\mathbb{P} V$ denotes the projective 3 -space of a fixed 4 -dimensional $k$-vector space $V$.

- $\mathcal{O}(d)$ denotes the invertible sheaf of degree $d$ on $\mathbb{P}_{3}, \quad \Omega^{p}$ the locally free sheaf of differential $p$-forms, $\omega=\Omega^{3}$ the dualizing sheaf.

- The terms vector bundle and locally free sheaf are used synonymously.

- If $\mathcal{F}$ is a coherent sheaf on $\mathbb{P}_{3}$ we use the abbreviations $\mathcal{F}(d)=\mathcal{F} \otimes \mathcal{O}(d)$, $H^{i} \mathcal{F}=H^{i}\left(\mathbb{P}_{3}, \mathcal{F}\right), \quad h^{i} \mathcal{F}=\operatorname{dim} H^{i} \mathcal{F}, \quad$ and $\quad m \mathcal{F}=k^{m} \otimes \mathcal{F}$, where for a vector space $E$ the symbol $E \otimes \mathcal{O}$ denotes the sheaf of sections of the trivial vector bundle with fibre $E$ and $E \otimes \mathcal{F}=(E \otimes \mathcal{O}) \otimes_{\mathcal{O}} \mathcal{F}$.

- We use the Euler sequence $0 \rightarrow \Omega^{1}(1) \rightarrow V^{\vee} \otimes \mathcal{O} \rightarrow \mathcal{O}(1) \rightarrow 0$ and the derived sequences in its Koszul complex $0 \rightarrow \Omega^{p}(p) \rightarrow \Lambda^{p} V^{\vee} \otimes \mathcal{O} \rightarrow \Omega^{p-1}(\mathrm{p}) \rightarrow 0$ without extra mentioning.

- A mathematical instanton bundle $\mathcal{E}$ on $\mathbb{P}_{3}$ is a rank-2 locally free sheaf with first Chern class $c_{1} \mathcal{E}=0$ and vanishing conditions $h^{0} \mathcal{E}=0$ and $h^{1} \mathcal{E}(-2)=0$. Since $c_{1} \mathcal{E}=0$ and $\operatorname{rank} \mathcal{E}=2$ the condition $h^{0} \mathcal{E}=0$ is the stability condition, see [3], [11]. 


\section{Multiple extensions and Koszul structures on lines}

1.1 By a multiple structure on a line $\ell$ in $\mathbb{P}_{3}$ we understand a subscheme $X$ of $\mathbb{P}_{3}$ whose underlying reduced subscheme is the line $\ell$. An $n$-fold extension of the line $\ell$ is defined by induction as follows. A 1 -fold extension is the line $\ell$ and an $n$-fold extension of $\ell$ is a subscheme $X$ which has a subscheme $X^{\prime} \subset X$ with exact sequence

$$
0 \rightarrow \mathcal{O}_{\ell} \rightarrow \mathcal{O}_{X} \rightarrow \mathcal{O}_{X^{\prime}} \rightarrow 0
$$

and s.t. $X^{\prime}$ is an $(n-1)$-fold extension of $\ell$. Then $X$ has multiplicity $n$, i.e. its Hilbert polynomial is $\mathcal{X O}_{X}(d)=n d+n$, and $X$ is Cohen-Macaulay. By adding resolutions of $\mathcal{O}_{\ell}$ and $\mathcal{O}_{X^{\prime}}$ in $\mathbb{P}_{3}$ we obtain a resolution

$$
0 \rightarrow n \mathcal{O}(-2) \stackrel{A^{\prime}}{\rightarrow} 2 n \mathcal{O}(-1) \stackrel{A}{\rightarrow} n \mathcal{O} \rightarrow \mathcal{O}_{X} \rightarrow 0 .
$$

It is easy to see by the induction process that the matrices $A$ and $A^{\prime}$ can be given the following triangular block form

$$
A^{\prime}=\left(\begin{array}{ccccc}
Z^{\prime} & & & & \\
A_{21}^{\prime} & Z^{\prime} & & & \\
\vdots & \ddots & \ddots & & \\
\vdots & & \ddots & \ddots & \\
A_{n 1}^{\prime} & \cdots & \cdots & A_{n, n-1}^{\prime} & Z^{\prime}
\end{array}\right) \quad A=\left(\begin{array}{ccccc}
Z & & & & \\
A_{21} & Z & & & \\
\vdots & \ddots & \ddots & & \\
\vdots & & \ddots & \ddots & \\
A_{n 1} & \cdots & \cdots & A_{n, n-1} & Z
\end{array}\right)
$$

where the block matrices are as follows. For any two forms $x, y \in V^{\vee}$ we define their Koszul matrices by $K(x, y)=\left(\begin{array}{l}x \\ y\end{array}\right)$ and $K^{\prime}(x, y)=(-y, x)$. We choose a decomposition $V^{\vee}=H^{0} \mathcal{I}_{\ell}(1) \oplus H$ with $H \cong H^{0} \mathcal{O}_{\ell}(1)$. Then $Z=K\left(z_{2}, z_{3}\right)$ for a basis $z_{2}, z_{3} \in H^{0} \mathcal{I}_{\ell}(1)$ and $A_{\mu \nu}=K\left(a_{\mu \nu}, b_{\mu \nu}\right)$ for some $a_{\mu \nu}, b_{\mu \nu} \in H$, and $Z^{\prime}, A_{\mu \nu}^{\prime}$ are the corresponding second Koszul matrices. In [10], $\S 5, n$-fold extension sheaves $\mathcal{L}$ of $\mathcal{O}_{\ell}(1)$ had been considered. If $\mathcal{L}=\mathcal{O}_{X}(1)$ then $\mathcal{L} \otimes \mathcal{O}_{\ell}=\mathcal{O}_{\ell}(1)$ is locally free and $\mathcal{L}$ is generated by two sections, using Nakayama's Lemma. From [10], Proposition 5.7. we obtain

Propositition 1.2 Let $X$ be an $n$-fold extension of $\ell$. Then $\mathcal{O}_{X}$ has a resolution

$$
0 \rightarrow n \mathcal{O}(-2) \stackrel{A^{\prime}}{\rightarrow} 2 n \mathcal{O}(-1) \stackrel{A}{\rightarrow} n \mathcal{O} \rightarrow \mathcal{O}_{X} \rightarrow 0
$$

where $A$ and $A^{\prime}$ are given as

$$
A^{\prime}=\left(\begin{array}{cccccc}
Z^{\prime} & & & & & \\
W^{\prime} & Z^{\prime} & & & & \\
A_{3}^{\prime} & W^{\prime} & Z^{\prime} & & & \\
\vdots & \ddots & \ddots & \ddots & & \\
\vdots & & \ddots & \ddots & \ddots & \\
A_{n}^{\prime} & \cdots & \cdots & A_{3}^{\prime} & W^{\prime} & Z^{\prime}
\end{array}\right) \quad A=\left(\begin{array}{ccccccc}
Z & & & & & \\
W & Z & & & & \\
A_{3} & W & Z & & & \\
\vdots & \ddots & \ddots & \ddots & & \\
\vdots & & \ddots & \ddots & \ddots & \\
A_{n} & \cdots & \cdots & A_{3} & W & Z
\end{array}\right)
$$

in which $Z=K\left(z_{2}, z_{3}\right)$ as before, $W=K\left(z_{0}, z_{1}\right)$ for a basis $z_{0}, z_{1} \in H \cong H^{0} \mathcal{O}_{\ell}(1)$ and $A_{\mu}=K\left(a_{\mu}, b_{\mu}\right)$ for some $a_{\mu}, b_{\mu} \in H$. 
Definition $1.3 \quad$ a) If $X$ is an $n$-fold extension of a line $\ell$ we also call $X$ or $\mathcal{O}_{X}$ a Koszul structure of multiplicity $n$ or an $n$-Koszul structure on $\ell$.

b) As in [5] a Cohen-Macaulay multiple structure $X$ on $\ell$ is called primitive if it is locally contained in a smooth surface.

Propositition 1.4 Ann-Koszul structure $X$ on $\ell$ is primitive and has dualizing sheaf $\omega_{X}=\mathcal{O}_{X}(-2)$. In particular $X$ is a locally complete intersection (l.c.i.).

Proof: By the resolution type $X$ must be Cohen-Macaulay. Its ideal contains the Fitting ideal of $n$-minors of the matrix $A$ in 1.2. It contains $n$-forms of the form

$$
z_{0}^{\mu} z_{1}^{\nu}\left(z_{0} z_{3}-z_{1} z_{2}\right)+f_{\mu \nu}, \quad \mu+\nu=n-2,
$$

where $f_{\mu \nu} \in I_{\ell}^{2}$, where $I_{\ell}$ is the ideal of $\ell$ in the coordinate ring.

If $z_{0} \neq 0$ then the local ideal of $X$ contains

$$
z_{3}-z_{1} z_{2}+f_{n-2,0}
$$

and if $z_{1} \neq 0$ the local ideal of $X$ contains

$$
z_{2}-z_{0} z_{3}+f_{0, n-2} .
$$

Since both functions define locally a smooth surface, $X$ is primitive. Applying the functor $\mathcal{H o m}_{\mathcal{O}}(-, \omega)$ to the resolution of $\mathcal{O}_{X}$, where $\omega$ is the dualizing sheaf $\Omega^{3}$ of $\mathbb{P}_{3}$, we obtain by $\omega_{X}=\mathcal{E} x t_{\mathcal{O}}^{2}\left(\mathcal{O}_{X}, \omega\right)$ and $\mathcal{E} x t_{\mathcal{O}}^{i}\left(\mathcal{O}_{X}, \omega\right)=0$ for $i \neq 2$ and by the self-duality, up to a symplectic form, of the resolution that $\omega_{X} \cong \mathcal{O}_{X}(-2)$.

Remark 1.5 The matrices $A_{\mu}, \mu \geq 2$, in the resolution of a Koszul structure yield moduli for fixed $n$. It is easy to see that $X$ is contained in the quadric

$$
z_{0} z_{3}-z_{1} z_{2}=0 \text { if and only if } A_{\mu}=0 \text { for } \mu \geq 2
$$

This is the case if the corresponding $n$-instanton bundle $\mathcal{E}$ satisfies $h^{0} \mathcal{E}(1)=2$, see $[6], \S 3$.

Remark 1.6 For an n-Koszul structure $X$ there are extension sequences

$$
0 \rightarrow \mathcal{O}_{X_{m}} \rightarrow \mathcal{O}_{X} \rightarrow \mathcal{O}_{X_{n-m}} \rightarrow 0
$$

for any $1 \leq m<n$ where $X_{m}$ and $X_{n-m}$ are again Koszul structures of multiplicity $m$ and $n-m$. This follows directly from the shape of the resolution matrices.

\subsection{The infinitesimal filtration of an $n$-Koszul structure}

Let $\ell^{(m)}$ denote the $\mathrm{m}$-th infinitesimal neighborhood of $\ell$ with ideal sheaf $\mathcal{I}_{\ell}^{m+1}, m \geq 0$. We consider the filtration $\ell=Y_{0} \subset Y_{1} \subset \ldots \subset Y_{n-1}=X$ of an $n$-Koszul structure $X$, where $Y_{m}=X \cap \ell^{(m)}$.

Since $\mathcal{I}_{X}$ contains $\mathcal{I}_{\ell}^{n}$, as follows directly by computing the Fitting ideal of $A$, we have $X \subset \ell^{(n-1)}$. By [5], 22 , the $Y_{\mu}$ are again primitive structures on $\ell$ of multiplicity $\mu+1$, and the embeddings $Y_{\mu-1} \hookrightarrow Y_{\mu}$ are described by exact sequences

$$
0 \rightarrow \mathcal{L}^{\otimes \mu} \rightarrow \mathcal{O}_{Y_{\mu}} \rightarrow \mathcal{O}_{Y_{\mu-1}} \rightarrow 0
$$

where $\mathcal{L}$ is an invertible sheaf on the reduced line $\ell$. 
Lemma 1.8 Let $X$ be an $n$-Koszul structure on $\ell$. Then the intersections $Y_{\mu}$ are again Koszul structures on $\ell$ and $\mathcal{L}=\mathcal{O}_{\ell}$.

Proof: Since $X$ is an $n$-fold extension there is an exact sequence

$$
0 \rightarrow \mathcal{O}_{\ell} \rightarrow \mathcal{O}_{X} \rightarrow \mathcal{O}_{X^{\prime}} \rightarrow 0
$$

where $X^{\prime}$ is an $(n-1)$-Koszul structure, $X^{\prime} \subset X$. Therefore $\mathcal{I}_{\ell}^{n-1} \subset \mathcal{I}_{X^{\prime}}$ and hence $\mathcal{I}_{Y_{n-2}}=\mathcal{I}_{X}+\mathcal{I}_{\ell}^{n-1} \subset \mathcal{I}_{X^{\prime}}$. From this we have an exact sequence

$$
0 \rightarrow \mathcal{C} \rightarrow \mathcal{O}_{Y_{n-2}} \rightarrow \mathcal{O}_{X^{\prime}} \rightarrow 0
$$

in which the kernel $\mathcal{C}$ must be a 0 -dimensional sheaf since both $Y_{n-2}$ and $X^{\prime}$ have multiplicity $n-1$. But since $Y_{n-2}$ is Cohen-Macaulay, $\mathcal{C}=0$, i.e. $Y_{n-1}=X^{\prime}$. Now we can finish the proof by induction on $n$.

Corollary $1.9 \quad$ 1) An n-Koszul structure $X$ has a unique filtration $\ell=X_{1} \subset \ldots \subset$ $X_{n}=X$ by $\mu$-Koszul structures $X_{\mu}=X \cap \ell^{(\mu+1)}$ with exact sequences

$$
0 \rightarrow \mathcal{O}_{\ell} \rightarrow \mathcal{O}_{X_{\mu+1}} \rightarrow \mathcal{O}_{X_{\mu}} \rightarrow 0
$$

2) The $n$-Koszul structures on $\ell$ are exactly the primitive structures of type $\mathcal{O}_{\ell}$ in the sense of [5],2.1

\subsection{Conormal sequence}

Let $X$ be an $n$-Koszul structure. By Lemma 1.7 and [5], proposition 2.3, the canonical homomorphism $\mathcal{N}_{X}^{\vee} \otimes \mathcal{O}_{\ell} \rightarrow \mathcal{N}_{\ell}^{\vee}$ of the conormal sheaves has kernel and cokernel equal to $\mathcal{O}_{\ell}$, i.e. we have an exact sequence

$$
0 \rightarrow \mathcal{O}_{\ell} \rightarrow \mathcal{N}_{X}^{\vee} \otimes \mathcal{O}_{\ell} \rightarrow \mathcal{N}_{\ell}^{\vee} \rightarrow \mathcal{O}_{\ell} \rightarrow 0
$$

Since $\mathcal{N}_{\ell}^{\vee} \cong 2 \mathcal{O}_{\ell}(-1)$ the sheaf $\mathcal{N}_{X}^{\vee} \otimes \mathcal{O}_{\ell}$ is an extension of $\mathcal{O}_{\ell}(-2)$ by $\mathcal{O}_{\ell}$ on the reduced line. Since the group $\operatorname{Ext}_{\ell}^{1}\left(\mathcal{O}_{\ell}(-2), \mathcal{O}_{\ell}\right) \cong H^{1} \mathcal{O}_{\ell}(2)=0$, this extension is trivial. Therefore

$$
\mathcal{N}_{X}^{\vee} \otimes \mathcal{O}_{\ell} \cong \mathcal{O}_{\ell} \oplus \mathcal{O}_{\ell}(-2)
$$

Propositition 1.11 Let $X$ be an $n$-Koszul structure on $\ell$. Then $H^{1} \mathcal{N}_{X}^{\vee}(1)=0$.

Proof: By induction on $n$; the statement being trivial for $n=1$. For $n>1$ we are given exact sequences

$$
0 \rightarrow \mathcal{O}_{\ell} \rightarrow \mathcal{O}_{X} \rightarrow \mathcal{O}_{Y} \rightarrow 0
$$

and

$$
0 \rightarrow \mathcal{I}_{X} \rightarrow \mathcal{I}_{Y} \rightarrow \mathcal{O}_{\ell} \rightarrow 0
$$

with $Y$ an $(n-1)$-Koszul structure. Dualizing (7) and using $\omega_{X}=\mathcal{O}_{X}(-2), \quad \omega_{Y}=\mathcal{O}_{Y}(-2)$ and $\omega_{\ell}=\mathcal{O}_{\ell}(-2)$ we also obtain the exact sequence 


$$
0 \rightarrow \mathcal{O}_{Y} \rightarrow \mathcal{O}_{X} \rightarrow \mathcal{O}_{\ell} \rightarrow 0
$$

If we tensor (7) and (9) by the locally free $\mathcal{O}_{X^{-}}$module $\mathcal{N}_{X}^{\vee}$ we get the exact sequences

$$
\begin{gathered}
0 \rightarrow \mathcal{N}_{X}^{\vee} \otimes \mathcal{O}_{\ell} \rightarrow \mathcal{N}_{X}^{\vee} \rightarrow \mathcal{N}_{X}^{\vee} \otimes \mathcal{O}_{Y} \rightarrow 0 \\
0 \rightarrow \mathcal{N}_{X}^{\vee} \otimes \mathcal{O}_{Y} \rightarrow \mathcal{N}_{X}^{\vee} \rightarrow \mathcal{N}_{X}^{\vee} \otimes \mathcal{O}_{\ell} \rightarrow 0
\end{gathered}
$$

Tensoring (8) by $\mathcal{O}_{Y}$ gives us the exact sequence

$$
\mathcal{T} \operatorname{or}_{1}^{\mathcal{O}}\left(\mathcal{O}_{\ell}, \mathcal{O}_{Y}\right) \rightarrow \mathcal{N}_{X}^{\vee} \otimes \mathcal{O}_{Y} \rightarrow \mathcal{N}_{Y}^{\vee} \rightarrow \mathcal{O}_{\ell} \rightarrow 0
$$

Using the resolution of $\mathcal{O}_{Y}$ as a Koszul structure we easily find

$\mathcal{T}$ or $_{1}^{\mathcal{O}}\left(\mathcal{O}_{\ell}, \mathcal{O}_{Y}\right) \cong 2 \mathcal{O}_{\ell}(-1)$. Now we proceed calculating the group $H^{1} \mathcal{N}_{X}^{\vee}(1)$. From (10) for the Koszul structure $Y$ with substructure $Z$ and the induction hypothesis we get

$$
0=H^{1} \mathcal{N}_{Y}^{\vee}(1)=H^{1} \mathcal{N}_{Y}^{\vee} \otimes \mathcal{O}_{Z}(1)
$$

because $\mathcal{N}_{Y}^{\vee} \otimes \mathcal{O}_{\ell}=\mathcal{O}_{\ell} \oplus \mathcal{O}_{\ell}(-2)$. Hence from (11) for $Y$ we get the surjection

$$
H^{0} \mathcal{N}_{Y}^{\vee}(1) \rightarrow H^{0} \mathcal{N}_{Y}^{\vee} \otimes \mathcal{O}_{\ell}(1) \rightarrow 0
$$

Now split (12) into two exact sequences

$$
\begin{gathered}
2 \mathcal{O}_{\ell}(-1) \rightarrow \mathcal{N}_{X}^{\vee} \otimes \mathcal{O}_{X} \rightarrow \mathcal{A} \rightarrow \mathcal{O} \\
0 \rightarrow \mathcal{A} \rightarrow \mathcal{N}_{Y}^{\vee} \stackrel{\pi}{\rightarrow} \mathcal{O}_{\ell} \rightarrow 0
\end{gathered}
$$

The surjection $\pi$ factors through

$$
\mathcal{N}_{Y}^{\vee} \otimes \mathcal{O}_{\ell}=\mathcal{O}_{\ell} \oplus \mathcal{O}_{\ell}(-2) \rightarrow \mathcal{O}_{\ell}
$$

whose first component $\alpha$ must be nonzero, hence an isomorphism. From (15) we get

$$
H^{1} \mathcal{N}_{X}^{\vee} \otimes \mathcal{O}_{Y}(1)=H^{1} \mathcal{A}(1)
$$

and from (16) and (14) the exact diagram

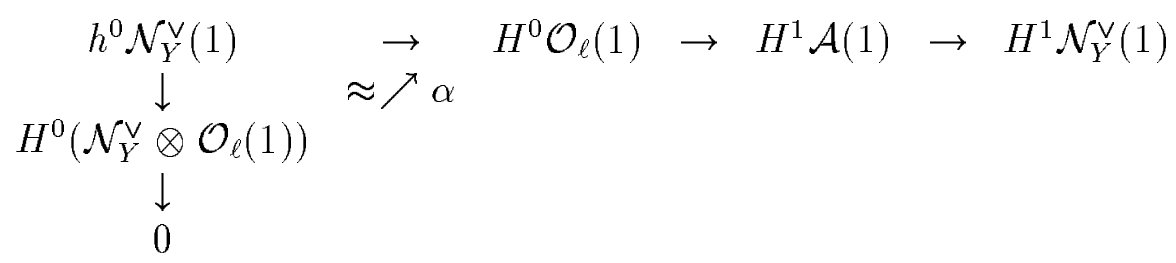

By induction hypothesis and the induced isomorphism $\alpha$ we conclude that $H^{1} \mathcal{A}(1)=$ 0 . By (10) again for $X$ we finally have $H^{1} \mathcal{N}_{X}^{\vee}(1)=H^{1} \mathcal{N}_{X}^{\vee} \otimes \mathcal{O}_{Y}(1)=H^{1} \mathcal{A}(1)=0$. 


\section{The Beilinson resolution}

For any coherent sheaf $\mathcal{F}$ on $\mathbb{P}_{3}$ there is a Beilinson complex

$$
0 \rightarrow C^{-3}(\mathcal{F}) \rightarrow C^{-2}(\mathcal{F}) \rightarrow \ldots \rightarrow C^{0}(\mathcal{F}) \rightarrow \ldots \rightarrow C^{3}(\mathcal{F}) \rightarrow 0
$$

which is exact except at $C^{0}(\mathcal{F})$ and has $\mathcal{F}$ as cohomology at $C^{0}(\mathcal{F})$, see [1]. The sheaves of the complex are given by

$$
C^{p}(\mathcal{F})=\bigoplus_{i-j=p} H^{i}\left(\mathcal{F} \otimes \Omega^{j}(j)\right) \otimes \mathcal{O}(-j)
$$

If $X$ is a 1-dimensional subscheme the only nonzero terms for $\mathcal{O}_{X}(-1)$ are

$$
\begin{array}{rlll}
C^{-3}=H^{0}\left(E_{X}^{3}\right) \otimes \mathcal{O}(-3) & & \\
C^{-2}=H^{0}\left(E_{X}^{2}\right) \otimes \mathcal{O}(-2) & \oplus H^{1}\left(E_{X}^{3}\right) \otimes \mathcal{O}(-3) \\
C^{-1}=H^{0}\left(E_{X}^{1}\right) \otimes \mathcal{O}(-1) & \oplus H^{1}\left(E_{X}^{2}\right) \otimes \mathcal{O}(-2) \\
C^{0}=H^{0}\left(E_{X}^{0}\right) \otimes \mathcal{O} & \oplus \begin{array}{l}
H^{1}\left(E_{X}^{1}\right) \otimes \mathcal{O}(-1) \\
C^{1}=
\end{array} & & H^{1}\left(E_{X}^{0}\right) \otimes \mathcal{O}
\end{array}
$$

where $E_{X}^{j}=\Omega^{j}(j-1) \otimes \mathcal{O}_{X}$. The following Lemma is a direct consequence.

Lemma 2.1 Let $X$ be a multiple structure on a line $\ell$. Then the following conditions are equivalent:

(i) $\mathcal{O}_{X}$ has a resolution $0 \rightarrow n \mathcal{O}(-2) \stackrel{B}{\rightarrow} 2 n \mathcal{O}(-1) \stackrel{A}{\rightarrow} n \mathcal{O} \rightarrow \mathcal{O}_{X} \rightarrow 0$

(ii) $h^{0} E_{X}^{j}=0$ and $h^{1} E_{X}^{3}=n, \quad h^{1} E_{X}^{2}=2 n, \quad h^{1} E_{X}^{1}=n, \quad h^{1} E_{X}^{0}=0$.

The conditions in 2.1 do not yet imply that $\mathcal{O}_{X}$ is a Koszul structure. The additional condition needed for that is selfduality.

Lemma 2.2 Let a multiple structure $X$ on a line $\ell$ satisfy the condition of Lemma 2.1. Then the following conditions are equivalent:

(i) The resolution $(B, A)$, is self-dual, i.e. $(B, A)$ is isomorphic to $\left(A^{t}, B^{t}\right)$

(ii) $\omega_{X} \cong \mathcal{O}_{X}(-2)$

Proof: Since $X$ is Cohen-Macaulay of dimension 1 the dual of the above resolution of $\mathcal{O}_{X}$ with respect to $\mathcal{H o m}_{\mathcal{O}}(-, \omega)$ gives us the resolution

$$
0 \rightarrow n \mathcal{O}(-4) \stackrel{A^{t}}{\rightarrow} 2 n \mathcal{O}(-3) \stackrel{B^{t}}{\rightarrow} n \mathcal{O}(-2) \rightarrow \omega_{X} \rightarrow 0 .
$$

Therefore (i) implies (ii). If conversely $\omega_{X} \cong \mathcal{O}_{X}(-2)$ then the two Beilinson resolutions (which are determined by the cohomology of the sheaves) must be isomorphic. Now we can prove 
Propositition 2.3 Any multiple structure $X$ on a line $\ell$ with a resolution

$$
0 \rightarrow n \mathcal{O}(-2) \rightarrow 2 n \mathcal{O}(-1) \rightarrow n \mathcal{O} \rightarrow \mathcal{O}_{X} \rightarrow 0
$$

and satisfying $\omega_{X}=\mathcal{O}_{X}(-2)$ is an $n$-Koszul structure.

Proof: By Lemma 2.1 the given resolution is the Beilinson resolution. It shows that $\mathcal{O}_{X}$ is Cohen-Macaulay. Since $\ell \subset X$ we have an exact sequence

$$
0 \rightarrow \mathcal{J} \rightarrow \mathcal{O}_{X} \rightarrow \mathcal{O}_{\ell} \rightarrow 0
$$

whose dual becomes

$$
0 \rightarrow \omega_{\ell} \rightarrow \omega_{X} \rightarrow \mathcal{E} x t_{\mathcal{O}}^{2}(\mathcal{J}, \omega) \rightarrow 0 .
$$

Twisting by 2 and using $\omega_{X}(2) \cong \mathcal{O}_{X}$, we get the exact sequence

$$
0 \rightarrow \mathcal{O}_{\ell} \rightarrow \mathcal{O}_{X} \rightarrow \mathcal{O}_{Y} \rightarrow 0
$$

in which $\mathcal{O}_{Y}$ is the quotient structure. We proceed now by induction on the multiplicity. For this it is enough to show that $Y$ again has a Beilinson resolution

$$
0 \rightarrow(n-1) \mathcal{O}(-2) \rightarrow(2 n-2) \mathcal{O}(-1) \rightarrow(n-1) \mathcal{O} \rightarrow \mathcal{O}_{Y} \rightarrow 0
$$

and satisfies $\omega_{Y}=\mathcal{O}_{Y}(-2)$.

To show that we first remark that $Y$ is again Cohen-Macaulay: The dual of (18) becomes

$$
0 \rightarrow \omega_{Y}(2) \rightarrow \mathcal{O}_{X} \stackrel{\pi}{\rightarrow} \mathcal{O}_{\ell} \rightarrow \mathcal{E} x t_{\mathcal{O}}^{3}\left(\mathcal{O}_{Y}, \omega\right) \rightarrow 0
$$

and since $\pi$ is the original surjection, the sheaf $\mathcal{E} x t^{3}$ is zero, which implies that $\mathcal{O}_{Y}$ has no 0 -dimensional torsion. We also have the exact sequence

$$
0 \rightarrow \omega_{Y}(2) \rightarrow \mathcal{O}_{X} \rightarrow \mathcal{O}_{\ell} \rightarrow 0
$$

Sequence (18) yields the exact sequences

$$
0 \rightarrow E_{\ell}^{j} \rightarrow E_{X}^{j} \rightarrow E_{Y}^{j} \rightarrow 0
$$

where as above $E^{j}=\Omega^{j}(j-1)$. Since $\Omega^{1}(1) \otimes \mathcal{O}_{\ell}=\Omega_{\ell}^{1}(1) \oplus 2 \mathcal{O}_{\ell}$ we obtain

$$
E_{\ell}^{j} \cong\left\{\begin{array}{cl}
\mathcal{O}_{\ell}(-1) & j=0 \\
\mathcal{O}_{\ell}(-2) \oplus 2 \mathcal{O}_{\ell}(-1) & j=1 \\
2 \mathcal{O}_{\ell}(-2) \oplus \mathcal{O}_{\ell}(-1) & j=2 \\
\mathcal{O}_{\ell}(-2) & j=3 .
\end{array}\right.
$$

Since $h^{1} E_{\ell}^{0}=0$ we get $h^{0} E_{Y}^{0}=h^{0} \mathcal{O}_{Y}(-1)=0$. If $z$ is a linear form, which is not a zero divisor for $\mathcal{O}_{\ell}$ we get injections $\mathcal{O}_{Y}(-d-1) \hookrightarrow \mathcal{O}_{Y}(-d)$ for $d \geq 0$, because $\mathcal{O}_{Y}$ is Cohen-Macaulay. Therefore $h^{0} \mathcal{O}_{Y}(-d)=0$ for $d \geq 0$. This implies $h^{0} E_{Y}^{j}=0$, using the standard Koszul resolutions of $\Omega^{j}$. Now we get the exact sequences

$$
0 \rightarrow H^{1} E_{\ell}^{j} \rightarrow H^{1} E_{X}^{j} \rightarrow H^{1} E_{Y}^{j} \rightarrow 0 .
$$

Since $h^{1} E_{X}^{0}=0$, also $h^{1} E_{Y}^{0}=0$. On the other hand $h^{1} E_{\ell}^{1}=1, h^{1} E_{\ell}^{2}=2, h^{1} E_{\ell}^{3}=1$. Hence $h^{1} E_{Y}^{1}=n-1, h^{1} E_{Y}^{2}=2 n-2, h^{1} E_{Y}^{3}=n-1$. By Lemma $2.1 \mathcal{O}_{Y}$ has a resolution (19). Dualizing this we get a resolution

$$
0 \rightarrow(n-1) \mathcal{O}(-2) \rightarrow(2 n-2) \mathcal{O}(-1) \rightarrow(n-1) \mathcal{O} \rightarrow \omega_{Y}(2) \rightarrow 0
$$


In order to show that $\omega_{Y} \cong \mathcal{O}_{Y}(-2)$ we let $\mathcal{L}$ denote the torsionfree part of $\omega_{Y}(2) \otimes \mathcal{O}_{\ell}$, such that

$$
\omega_{Y}(2) \otimes \mathcal{O}_{\ell}=\mathcal{T} \oplus \mathcal{L} .
$$

$\mathcal{L}$ is a vector bundle on $\ell$ which is generated by global sections coming from $\omega_{Y}(2)$, hence $\mathcal{L}=\oplus \mathcal{O}\left(a_{i}\right)$ with $a_{i} \geq 0$. On the other hand sequence (20) gives us a surjection $\mathcal{T}_{\text {or }}\left(\mathcal{O}_{\ell}, \mathcal{O}_{\ell}\right) \rightarrow \omega_{Y}(2) \otimes \mathcal{O}_{\ell}$, hence a surjection $2 \mathcal{O}_{\ell}(-1) \rightarrow \mathcal{L}$. It follows that $\mathcal{L}=$ $\mathcal{O}_{\ell}(a)$ is of rank 1 . Now let $p \in \ell \backslash \operatorname{Supp}(\mathcal{T})$. Since $\mathcal{O}_{\ell}(a)$ is globally generated by induced sections of $\omega_{Y}(2)$, there is one section $\mathcal{O} \rightarrow \omega_{Y}(2)$ which generates $\mathcal{O}_{\ell}(a)$ at $p$ under

$$
\mathcal{O} \rightarrow \omega_{Y}(2) \rightarrow \omega_{Y}(2) \otimes \mathcal{O}_{\ell} \rightarrow \mathcal{O}_{\ell}(a) .
$$

By Nakayama's Lemma this section generates $\omega_{Y}(2)$ at $p$. Therefore $\mathcal{O} \rightarrow \omega_{Y}(2)$ is generically surjective. It induces a sequence

$$
0 \rightarrow \mathcal{C}^{\prime} \rightarrow \mathcal{O}_{Y} \rightarrow \omega_{Y}(2) \rightarrow \mathcal{C}^{\prime \prime} \rightarrow 0
$$

where $\mathcal{C}^{\prime \prime}$ is 0 -dimensional. Since both $\mathcal{O}_{Y}$ and $\omega_{Y}(2)$ have the same Hilbert polynomial, also $\mathcal{C}^{\prime}$ is 0 -dimensional, hence $\mathcal{C}^{\prime}=0$. We thus have shown that $\mathcal{O}_{Y} \cong \omega_{Y}(2)$, which completes the proof of proposition 2.3 . 


\section{Instanton bundles with one linear section}

A mathematical instanton bundle on $\mathbb{P}_{3}$ is a stable rank 2 vector bundle $\mathcal{E}$ with first Chern class $c_{1} \mathcal{E}=0$ and vanishing condition $h^{1} \mathcal{E}(-2)=0$, see [4]. The stability condition implies $n=c_{2} \mathcal{E}>0$. It is well-known that $\mathcal{E}$ is the cohomology of a Beilinson complex

$$
0 \rightarrow n \Omega^{3}(3) \stackrel{M}{\rightarrow} n \Omega^{1}(1) \stackrel{B}{\rightarrow}(2 n-2) \mathcal{O} \rightarrow 0
$$

in which $M$ and $B$ are induced by linear maps

$$
k^{n} \stackrel{M}{\rightarrow} k^{n} \otimes \wedge^{2} V, \quad k^{n} \stackrel{B}{\rightarrow} k^{2 n-2} \otimes V .
$$

The conditions for $M, B$ to define an instanton bundle are:

(i) $M$ is symmetric

(ii) the induced sequence

$$
k^{n} \otimes V \stackrel{\wedge M}{\rightarrow} k^{n} \otimes \wedge^{3} V \stackrel{\wedge B}{\rightarrow} k^{2 n-2} \otimes \wedge^{4} V \rightarrow 0
$$

is exact

(iii) $k^{2 n-2} \stackrel{B^{t}}{\longrightarrow} k^{n} \otimes V$ satisfies $\operatorname{Im}\left(B^{t}\right) \cap\left(k^{n} \otimes v\right)=0$ for any nonzero $v \in V$

see [6], section 1 . We let $M I(n)$ denote the open subscheme of the Maruyama scheme $M(2 ; 0, n, 0)$ of all semi-stable coherent sheaves on $\mathbb{P}_{3}$ of rank 2 and Chern classes $\left(c_{1}, c_{2}, c_{3}\right)=(0, n, 0)$ whose closed points are the isomorphism classes of mathematical instanton bundles. Up to now it is not known whether $M I(n)$ is smooth and irreducible for all $n$. $M I(n)$ is smooth at $\mathcal{E}$ if $\operatorname{Ext}^{2}(\mathcal{E}, \mathcal{E})=0$. There are reasons to believe that the stronger condition $\operatorname{Ext}^{2}(\mathcal{E}, \mathcal{E}(-1))=0$ holds for any $\mathcal{E} \in M I(n)$. Indeed this is true for the so-called special ' $t$ Hooft instanton bundles characterized by $h^{0} \mathcal{E}(1)=2$, see [6]. This was shown in [8], or can easily be derived from the normal form of $B$ in [6]. We are going to show that $\operatorname{Ext}^{2}(\mathcal{E}, \mathcal{E}(-1))=0$ also holds for any $\mathcal{E} \in M I(n)$ satisfying $h^{0} \mathcal{E}(1)=1$. Note that by $[6] h^{0} \mathcal{E}(1) \leq 2$ for any $\mathcal{E} \in M I(n)$. In the following we assume $n \geq 3$, since for $n=2$ always $h^{0} \mathcal{E}(1)=2$.

3.1 By the general Serre construction, see [7], rank 2 bundles can be constructed on $\mathbb{P}_{3}$ from l.c.i. intersection curves $X$ s.t. the dualizing sheaf $\omega_{X}$ is the restriction of a line bundle on $\mathbb{P}_{3}$. In particular, if $X$ is an $n$-Koszul structure on a line $\ell$ with $\omega_{X}=\mathcal{O}_{X}(-2)$, we have

$$
\begin{aligned}
H^{0} \omega_{X}(2)=\operatorname{Hom}_{\mathcal{O}_{X}}\left(\mathcal{O}_{X}(-2), \omega_{X}\right) & =\operatorname{Ext}_{\mathcal{O}}^{2}\left(\mathcal{O}_{X}(-2), \omega_{\mathbb{P}_{3}}\right) \\
& =\operatorname{Ext}^{1}\left(\mathcal{I}_{X}(-2), \omega_{\mathbb{P}_{3}}\right) \\
& =\operatorname{Ext}^{1}\left(\mathcal{I}_{X}(1), \mathcal{O}(-1)\right)
\end{aligned}
$$


using Grothendieck duality. A section $s$ of $\omega_{X}(2)$ thus defines an extension $\mathcal{F}$,

$$
0 \rightarrow \mathcal{O}(-1) \rightarrow \mathcal{F} \rightarrow \mathcal{I}_{X}(1) \rightarrow 0
$$

which is locally free if $s$ defines an isomorphism $\mathcal{O}_{X} \cong \omega_{X}(2)$. If not, $\mathcal{F}$ will be singular along the zero scheme $Z(s) \subset X$, see 4 . for an example. In our case one easily verifies, using the resolution of $\mathcal{O}_{X}$, that

$$
c_{1} \mathcal{F}=0, c_{2} \mathcal{F}=n, c_{3} \mathcal{F}=0, h^{0} \mathcal{F}=0, h^{1} \mathcal{F}(-2)=0
$$

and $h^{0} \mathcal{F}(1)=1$. Hence, if $\mathcal{F}$ is a bundle it belongs to $M I(n)$. We can now prove the converse:

Propositition 3.2 Let $\mathcal{E}$ be an instanton bundle with second Chern class $n-1 \geq 3$ such that $h^{0} \mathcal{E}(1)=1$, and let $X$ be the zero scheme of the non-zero section $s$ of $\mathcal{E}(1)$. then

(i) $X_{\text {red }}$ is a disjoint union of lines $\ell_{1}, \ldots, \ell_{m}$;

(ii) $X$ is a disjoint union of Koszul structures $X_{1}, \ldots, X_{m}$ on $\ell_{1}, \ldots, \ell_{m}$ respectively.

Proof: 1) Since $h^{0} \mathcal{E}=0, X$ is a 1 -dimensional 1.c.i., and we are given the exact sequence

$$
0 \rightarrow \mathcal{O}(-2) \stackrel{s}{\rightarrow} \mathcal{E}(-1) \rightarrow \mathcal{O} \rightarrow \mathcal{O}_{X} \rightarrow 0
$$

Its dual sequence becomes

$$
0 \rightarrow \mathcal{O} \stackrel{s}{\rightarrow} \mathcal{E}(1) \rightarrow \mathcal{O}(2) \rightarrow \omega_{X} \rightarrow 0
$$

where we use $\mathcal{E} \cong \mathcal{E}^{\vee}$, which follows from $\wedge^{2} \mathcal{E} \cong \mathcal{O}$. Hence $\omega_{X}=\mathcal{O}_{X}(-2)$.

2) Statement (i) was proved in [6], 1.3, using the monad description of $\mathcal{E}$ as above. Therefore, $X$ is a disjoint union of possibly multiple structures $X_{\mu}$ on $\ell_{\mu}$. In order to prove (ii) we can calculate the dimensions of the cohomology groups of $\mathcal{O}_{X}$ from (22) using (21). By (21) (for $n-1$ )

$$
\begin{aligned}
h^{0} \mathcal{E}(d) & =0 \quad \text { for } d \leq 0 \\
h^{1} \mathcal{E}(d) & =0 \quad \text { for } d \leq-2 \\
h^{2} \mathcal{E}(d) & =0 \quad \text { for } d \geq-2 \\
h^{3} \mathcal{E}(d) & =0 \quad \text { for } d \geq-4 \\
h^{2} \mathcal{E}(-3) & =h^{1} \mathcal{E}(-1)=n-1 \\
h^{1} \mathcal{E} & =2 n-4 .
\end{aligned}
$$


From $(22)$ we get $H^{1} \mathcal{O}_{X}(-1)=H^{2} \mathcal{I}_{X}(-1)=0$ because $H^{2} \mathcal{E}(-2)=0$, and similarly $H^{0} \mathcal{O}_{X}(-1)=0$. From this it is easy to see that $H^{0} E_{X}^{j}=H^{0} \mathcal{O}_{X} \otimes \Omega^{j}(j-1)=0$ for $0 \leq j \leq 3$. It remains to calculate $h^{1} E_{X}^{j}$. We have the exact sequence from the Euler sequence

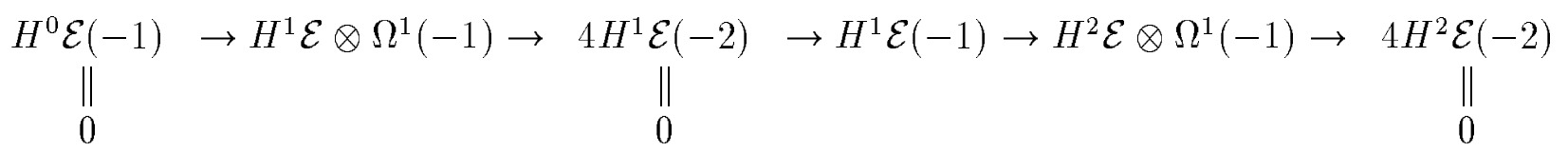

and from $(22)$

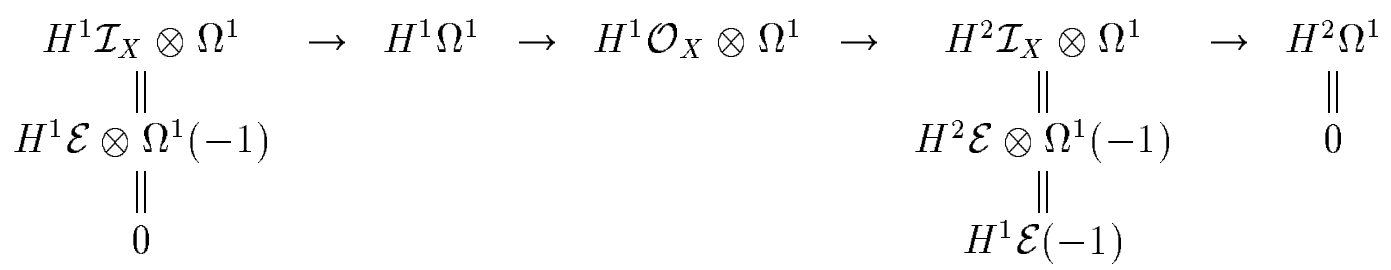

hence $h^{1} E_{X}^{1}=1+(n-1)=n$. Similarly, we obtain

$$
H^{1} E_{X}^{2}=H^{2} \mathcal{I}_{X} \otimes \Omega^{2}(1)=H^{2}\left(\mathcal{E} \otimes \Omega^{2}\right)
$$

with $h^{2}\left(\mathcal{E} \otimes \Omega^{2}\right)=2(n-1)+2=2 n, \quad$ and

$$
\begin{gathered}
H^{1} E_{X}^{3} \\
\| H^{2} \mathcal{E}(-3) \rightarrow H^{2} \mathcal{I}_{X} \otimes \Omega^{3}(2) \rightarrow H^{3} \Omega^{3} \rightarrow 0 .
\end{gathered}
$$

These data imply by Lemma 2.1 that $\mathcal{O}_{X}$ has a Beilinson resolution

$$
0 \rightarrow n \mathcal{O}(-2) \rightarrow 2 n \mathcal{O}(-1) \rightarrow n \mathcal{O} \rightarrow \mathcal{O}_{X} \rightarrow 0
$$

In order to show that each of the $X_{\mu}$ has a resolution of the same type we proceed as in the proof of Proposition 2.3. Assume that mult $\left(X_{1}\right) \geq 2$, say. There is a sequence

$$
0 \rightarrow \mathcal{O}_{\ell_{1}} \rightarrow \mathcal{O}_{X} \rightarrow \mathcal{O}_{Y} \rightarrow 0
$$

with $Y \subset X$ satisfying (i), (ii) of Proposition 2.1, and $Y=Y_{1} \dot{\cup} X_{2} \dot{\cup} \ldots \dot{\cup} X_{m}$.

By induction over $n$ we may assume that $Y_{1}, X_{2}, \ldots, X_{m}$ are Koszul structures. Then also $X_{1}$ is a Koszul structure. In particular, the above resolution of $\mathcal{O}_{X}$ can be replaced by the direct sum of the Beilinson resolutions of $\mathcal{O}_{X_{\mu}}$.

Theorem 3.3 Let $\mathcal{E}$ be an instanton bundle on $\mathbb{P}_{3}$. If $h^{0} \mathcal{E}(1)>0$ then $\operatorname{Ext} t^{2}(\mathcal{E}, \mathcal{E}(-1))$ $=\operatorname{Ext}^{2}(\mathcal{E}, \mathcal{E})=0$. 
Proof: By [6] $h^{0} \mathcal{E}(1) \leq 2$. If $h^{0} \mathcal{E}(1)=2$ this follows from [8]. If $h^{0} \mathcal{E}(1)=1$ we obtain an exact sequence (22) where $X=X_{1} \dot{\cup} \ldots \dot{\cup} X_{m}$ is a disjoint union of Koszul structures $X_{\mu}$ on lines $\ell_{\mu}$. By Proposition $1.8 H^{1} \mathcal{N}_{X}^{\vee}(1)=\oplus H^{1} \mathcal{N}_{X_{\mu}}^{\vee}(1)=0$, where $\mathcal{N}_{X}^{\vee}$ denotes the conormal sheaf of $X$.

The sequence (22) induces the exact sequence

$$
\operatorname{Ext}^{2}\left(\mathcal{I}_{X}(1), \mathcal{E}(-1)\right) \rightarrow \operatorname{Ext}^{2}(\mathcal{E}, \mathcal{E}(-1)) \rightarrow \underset{0}{H^{2} \mathcal{E}}
$$

On the other hand, we obtain

$$
\begin{array}{rlr} 
& \operatorname{Ext}^{2}\left(\mathcal{I}_{X}(1), \mathcal{E}(-1)\right) & \\
= & \operatorname{Ext}^{3}\left(\mathcal{O}_{X}, \mathcal{E}(-2)\right) & \text { since } H^{2} \mathcal{E}(-2)=H^{3} \mathcal{E}(-2)=0 \\
= & H^{1}\left(\mathcal{E} x t^{2}\left(\mathcal{O}_{X}, \mathcal{E}(-2)\right)\right. & \text { by Leray's spectral sequence } \\
= & H^{1}\left(\mathcal{E} x t^{2}\left(\mathcal{O}_{X}, \mathcal{O}(-2)\right) \otimes \mathcal{E}\right) & \text { since } \mathcal{E} \text { is locally free } \\
= & H^{1}\left(\mathcal{E} x t^{2}\left(\mathcal{O}_{X}(-2), \omega_{\mathbb{P}_{3}}\right) \otimes \mathcal{E}\right) & \\
=H^{1}\left(\omega_{X}(2) \otimes \mathcal{E}\right) & \text { by Grothendieck duality } \\
=H^{1}\left(\mathcal{E} \otimes \mathcal{O}_{X}\right) & \text { since } \omega_{X}(2)=\mathcal{O}_{X} .
\end{array}
$$

If we tensor sequence (22) by $\mathcal{O}_{X}$ we get the exact sequence

$$
\mathcal{O}_{X}(-1) \rightarrow \mathcal{E} \otimes \mathcal{O}_{X} \rightarrow \mathcal{N}_{X}^{\vee}(1) \rightarrow 0
$$

and this implies

$$
H^{1}\left(\mathcal{E} \otimes \mathcal{O}_{X}\right)=H^{1} \mathcal{N}_{X}^{\vee}(1)=0
$$

This proves the vanishing of $\operatorname{Ext}^{2}(\mathcal{E}, \mathcal{E}(-1))$. To show that also $\operatorname{Ext}^{2}(\mathcal{E}, \mathcal{E})=0$ we consider a plane $P \subset \mathbb{P}_{3}$ and the restriction sequence

$$
0 \rightarrow \mathcal{E}(-1) \rightarrow \mathcal{E} \rightarrow \mathcal{E} \otimes \mathcal{O}_{P} \rightarrow 0
$$

In the induced exact sequence

$$
\operatorname{Ext}^{2}(\mathcal{E}, \mathcal{E}(-1)) \rightarrow \operatorname{Ext}^{2}(\mathcal{E}, \mathcal{E}) \rightarrow \operatorname{Ext}^{2}\left(\mathcal{E}, \mathcal{E} \otimes \mathcal{O}_{P}\right)
$$

also the last group vanishes, because it is Serre-dual to $H^{0}\left(\mathcal{E} \otimes \mathcal{E} \otimes \mathcal{O}_{P}(-3)\right)=0$.

Since $E x t^{2}(\mathcal{E}, \mathcal{E})$ is the group of obstructions to smoothness of the Maruyama scheme at stable points, see [9] and [12], $\mathrm{MI}(\mathrm{n})$ is smooth at $\mathcal{E}$. 


\section{Extensions of Koszul structures and elemen- tary transformations}

In [10] $m$-fold extensions $\mathcal{L}$ of $\mathcal{O}_{\ell}(1)$ were used to construct sheaves in the boundary of $M I(n)$, called elementary transformations, as kernels in sequences $0 \rightarrow \mathcal{F} \rightarrow \mathcal{E} \rightarrow$ $\mathcal{L} \rightarrow 0$ where $\mathcal{E} \in M I(n-m)$. As remarked in 1.1 these $m$-fold extensions are nothing but $\mathcal{L}=\mathcal{O}_{X}(1)$ where $X$ is an $m$-Koszul structure. We remark here that the elementary transformation sequences arise naturally via Serre construction, 3.1, from extension sequences

$$
0 \rightarrow \mathcal{O}_{X^{\prime}} \rightarrow \mathcal{O}_{X} \rightarrow \mathcal{O}_{X^{\prime \prime}} \rightarrow 0
$$

given by the special form of the resolution of a Koszul structure $X$, where mult $\left(X^{\prime}\right)+$ $\operatorname{mult}\left(X^{\prime \prime}\right)=\operatorname{mult}(X)$. Given $(23)$ we get the exact sequence

$$
0 \rightarrow \mathcal{I}_{X} \rightarrow \mathcal{I}_{X^{\prime \prime}} \rightarrow \mathcal{O}_{X^{\prime}} \rightarrow 0
$$

and from this the diagram

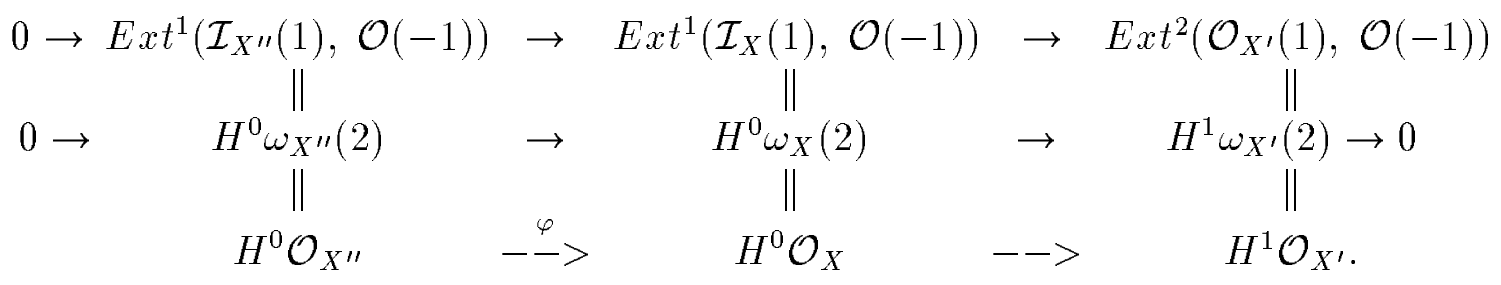

In this diagram the vertical isomorphisms are as in 3.1. Let $\xi=\varphi(1)$, where $\varphi$ is the induced homomorphism, and let $\mathcal{E}^{\prime \prime}$ and $\mathcal{F}$ be the corresponding extensions to 1 and $\xi$ respectively.

Since $\varphi$ corresponds to the pullback construction of extensions we get the exact diagram

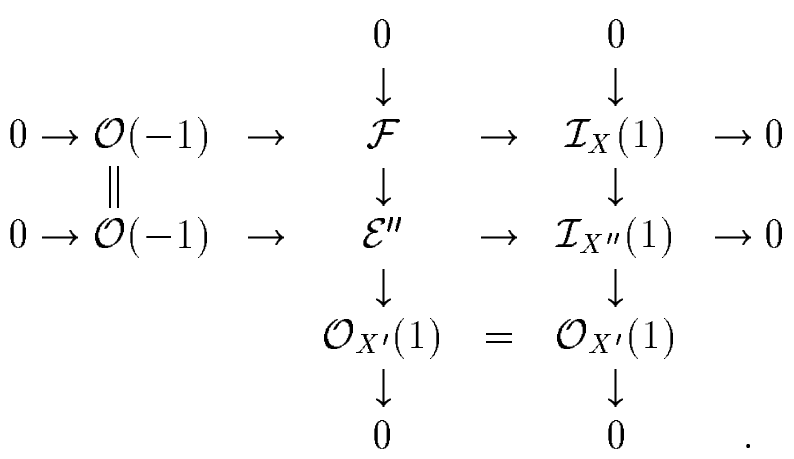

The sheaf $\mathcal{E}^{\prime \prime}$ is an instanton bundle with $c_{2} \mathcal{E}^{\prime \prime}=\operatorname{mult}\left(X^{\prime \prime}\right)-1$ by Serre construction, since the section 1 generates $\omega_{X}{ }^{\prime \prime}(2)$ everywhere. However, $\mathcal{F}$ is not locally free along $X^{\prime}$, since $\xi$ vanishes along $X^{\prime}$. Of course, this follows from the vertical sequence in the middle of the diagram, too.

The diagram shows that the section of $\mathcal{F}(1)$ given by it vanishes along $X$ as a section of $\mathcal{F}(1)$, but vanishes only along $X^{\prime \prime}$ as a section of $\mathcal{E}^{\prime \prime}(1)$. 


\section{$5 \quad$ Normal forms of monad arrows}

We close with a remark on normal forms of the right arrow $k^{n} \stackrel{B}{\rightarrow} k^{2 n-2} \otimes V$ of a monad (21) for an instanton bundle $\mathcal{E} \in M I(n)$ with $h^{0} \mathcal{E}(1)=1$. This matrix is nothing but a representation of the canonical homomorphism

$$
H^{1} \mathcal{E}(-1) \otimes V^{\vee} \rightarrow H^{1} \mathcal{E}
$$

Since $\mathcal{E}$ is obtained by the Serre construction from a disjoint union $X=X_{1} \cup \ldots \cup X_{m}$ of Koszul structures on lines, we have $H^{1} \mathcal{E}(-1)=H^{1} \mathcal{I}_{X}$ and $H^{1} \mathcal{E}=H^{1} \mathcal{I}_{X}(1)$. Therefore, a normal form of $B$ can be derived from the normal form of the resolutions (2) of the structures $\mathcal{O}_{X_{\mu}}$. For example, if $X$ is concentrated on one line $\ell$, spanned by $e_{0}, e_{1} \in V$, and $e_{2}, e_{3} \in V$ are complementary vectors, $B$ can be given the form

$$
\left(\begin{array}{ccccc}
E & & & & \\
F & \ddots & & & \\
B_{3} & \ddots & \ddots & & \\
\vdots & \ddots & \ddots & \ddots & \\
B_{n-1} & \cdots & B_{3} & F & E \\
\hline C_{n} & \cdots & \cdots & C_{2} & F
\end{array}\right)
$$

where $E, F, B_{\nu}, C_{\nu}$ are $1 \times 2$-matrices of vectors in $V$ given as $E=\left(e_{0}, e_{1}\right), F=$ $\left(e_{2}, e_{3}\right), B_{\nu}=\left(b_{\nu}, b_{\nu}^{\prime}\right)$ with $b_{\nu}, b_{\nu}^{\prime} \in \operatorname{Span}\left(e_{2}, e_{3}\right)$ and $C_{\nu}=\left(c_{\nu}, c_{\nu}^{\prime}\right)$ with arbitrary vectors $c_{\nu}, c_{\nu}^{\prime}$ in $V$. Note that the case $B_{\nu}=0, C_{\nu}=0$ gives us the normal form of $B$ if $h^{0} \mathcal{E}(1)=2$, see [6]. This is the case in which $X$ is contained in a quadric. 


\section{References}

[1] V. Ancona-G. Ottaviani, Canonical resolutions of sheaves on Schubert and Brieskorn varieties, (Preprint 1989)

[2] A. Beilinson, Coherent sheaves on $\mathbb{P}^{n}$ and problems of linear algebra, Funkt. Analiz. Prilozhenia 12, 68-69, 1978

[3] W. Barth, Some properties of stable rank 2 vector bundles on $\mathbb{P}_{n}$, Math. Ann. $226,125-150,1977$

[4] W. Barth-K. Hulek, Monads and moduli of vector bundles, manuscripta math. $25,323-347,1978$

[5] C. Banica - O. Forster, Multiple Structures on Space \& Curves in Contemporary Mathematics vol. 58, Proceedings of the Lefschetz Centennial Conference 1984, part I, AMS.

[6] W. Böhmer-G. Trautmann, Special instanton bundles and Poncelet curves, in Singularities, Representations of Algebras and Vector Bundles, Proceedings, Lambrecht, 1985.

[7] R. Hartshorne, Stable vector bundles of rank 2 on $\mathbb{P}_{3}$, Math. Ann. 238, 229-280, 1978.

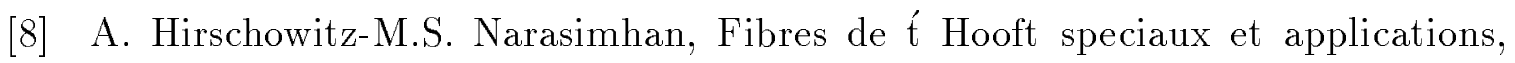
Proc. Nice Conf. 1981, Birkhäuser.

[9] M. Maruyama, Moduli of stable sheaves I \& II, J. Math. Kyoto Univ. 17, 91-126, $1977 \& 18,557-614,1978$.

[10] M. Maruayama - G. Trautmann, Limits of Instantons, Intern. Journal of Math. 3, 213-276, 1992 .

[11] Ch. Okonek - M. Schneider - H. Spindler, Vector bundles on complex projective spaces, Birkhäuser 1980 .

[12] J. Wehler, Moduli space and versal deformation of stable vector bundles, Revue Roum. de Math. Pure et Appl. 30, 69-78, 1985

Fachbereich Mathematik

Universität Kaiserslautern

D- 6750 Kaiserslautern

Email:

thomas@mathematik.uni-kl.de

trm@mathematik.uni-kl.de 